\title{
Electronic Structure and Absorption Spectrum of Disperse Red 1: Comparison of Computational Approaches
}

\author{
Jussi Ojanen* and Tapio T. Rantala*
}

Department of Physics, P.O.B. 692, FI-33101 Tampere University of Technology, Finland

\begin{abstract}
An azobenzene molecule Disperse Red 1 with strongly delocalised frontier orbitals has been studied with a number of density-functional theory (DFT) related approaches. The purpose is two-fold: to interpret observed photoabsorption and to compare the performance of various DFT-based approximations. The planarity of the vacuum conformation of the lower energy trans conformation is found to be significantly distorted when transformed to the higher energy cis conformation, and both isomers are found to absorb in the experimentally observed wavelengths in solutions. A common feature in both is essentially forbidden lowest energy absorption because of a negligible overlap of HOMO and LUMO due to symmetry unmatch. We find the time-dependent DFT to be the best approach for quantitative evaluation of photoabsorption energies and intensities, whereas Kohn-Sham eigenenergies and orbitals give good description only qualitatively. Isomerisation and dimerisation energetics have also been evaluated, and trans dimers have found to be up to $1 \mathrm{eV}$ per molecule more stable than single molecules.
\end{abstract}

\section{INTRODUCTION}

Azobenzene-containing materials are of immense interest for a variety of linear and nonlinear optical applications [13]. Their fascinating properties are largely based on reversible photoisomerisation of the azobenzene molecule: Upon irradiation with UV or visible light, the thermally stable trans configuration may undergo a transformation to the metastable cis configuration, which reverts back to the trans state either thermally or through electronic excitation. The optical and photophysical properties of azobenzene molecules depend strongly on the substituents $[4,5]$. In optical applications perhaps the most widely used are the "pseudostilbene"-type molecules, where the azobenzene is substituted with electron-donor and electron-acceptor groups. Pseudostilbenes are characterised by short lifetime of the cis isomer, a property that is useful in optical information storage and hologrpahic recording [6]. Moreover, the asymmetric electron distribution gives rise to large groundstate dipole moment and hyperpolarizabilities, making them suitable for nonlinear optical applications [7].

For practical device applications, the azobenzene moieties have to be incorporated in a solid matrix. The most attractive alternative is to use polymers because of their inexpensive preparation and easy fabrication into devices [8]. However, as it is often required to use high chromophore concentration to obtain high response to the optical fields, intermolecular interactions between the dyes have to be taken into account. Such interactions change the spectroscopic properties of the material and typically also suppress the overall response of the system $[9,10]$. Such interactions are especially severe for high-dipole-moment chromophores, and to prevent them, and on the other hand,

*Address correspondence to these author at the Department of Physics, P.O.B. 692, FI-33101 Tampere University of Technology, Finland; E-mails: Jussi.Ojanen@tut.fi or Tapio.Rantala@tut.fi to predict their effect on the response of the material system is of great importance, especially in case of nonlinear optical materials [11].

An inexpensive and thus attractive method for materials design is computational modelling. Because of its sophisticated nature, however, the methods available in practice are approximative. Therefore, also in case of quantum mechanics, though long since being highly useful, they are still calling for further development. In practice it requires, among other things, comparison of different approximations to gain higher level understanding. It would be particularly desirable to have accurate $a b$ initio methods, DFT as an example, because of their universality. However, calculation of the excitation energy, photoisomerisation, or intermolecular interactions of azobenzene chromophores within chemically satisfying accuracy is a challenging task. Aspects, such as restrictions on the feasible system size limiting the inclusion of the effects of the environment, statistical phenomena like the temperature, and the non-local nature of quantum mechanics especially is very demanding to account for but approximately. Fortunately, a lot of useful information can be deduced without including these phenomena exactly. Several aspects of azobenzene systems can and some have been studied by theoretical and computational modelling, e.g. $[12,13]$. The semiempirical methods sometimes reach the desired quality, but with the cost of eventually compromising the generality.

In this work, we have studied the properties of 4-[N-ethylN-(2-hydroxyethyl)]amino-4'-nitro-azobenzene, known as Disperse Red $1^{1}$ (DR1, see Figs. (1) and (2)), a common pseudostilbene-type molecule in a variety of optical applications. Despite various experimental studies [9, 14-18]

\footnotetext{
${ }^{1}$ The molecule's shape resembles the human figure (see Fig. (2)) so much that can't resist choosing the nomenclature accordingly (head, legs, toes, heel etc. e.g. in Figs. (2), (7) and (8)).
} 
containing DR1-like molecules, only few theoretical studies [19] have been published. We start by presenting a comprehensive DFT-study on trans -DR1, continue by investigation of the photoisomerisation in a more explorative fashion and finish with an extensive discussion on dimerisation of DR1. The calculated results are compared to experimental results. A central part of this work is also to compare different DFT-based approximations.

trans-DR1

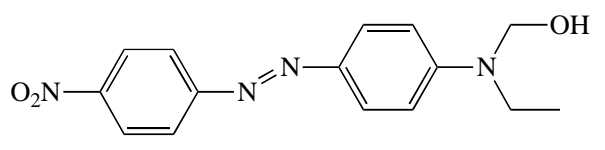

cis-DR1

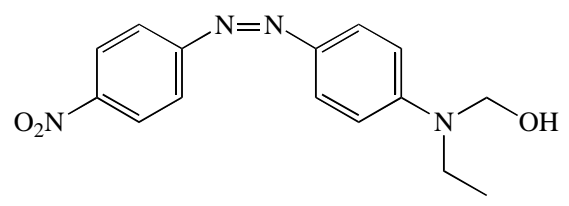

Fig. (1). The chemical formula of DR1.
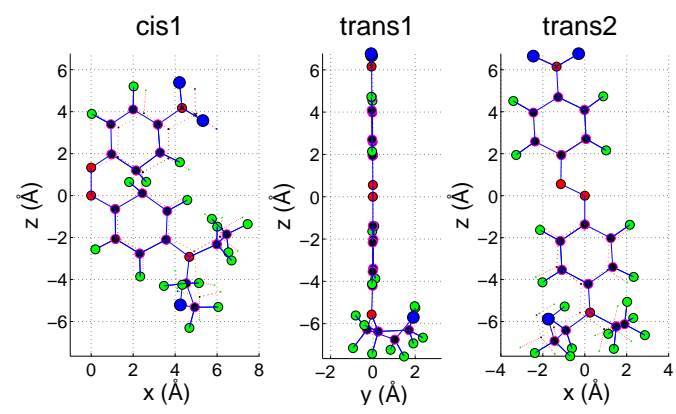

Fig. (2). The calculated minimum energy molecular structure of cis (left) and trans (middle and right) isomers of DR1: Optimised conformations with LDA functional (PWLDA as implemented in Turbomole). The red dotted line is the conformation obtained from the relaxed trans1 conformation by the appropriate rotation wrt a bond ( $\mathrm{N}=\mathrm{N}$ in trans to cis and $\mathrm{C}-\mathrm{N}$ in 1 to 2 transformation) before relaxation. The figure demonstrates e.g. the non-planarity of cis isomer and opening mechanism of the angles $\mathrm{C}-\mathrm{N}=\mathrm{N}-\mathrm{C}$ in trans to cis transformation (see Table 1). The suffices 1 and 2 in trans and cis refer to the location of the oxygen atom in the 'legs' (left and right respectively). See footnote 1 .

\section{ELECTRONIC STRUCTURE CALCULATIONS}

\section{A. Approximations to Density Functional Theory}

Evaluation of physical properties here relies largely on standard Kohn-Sham Density Functional Theory. Though formally exact and existing, nevertheless in practice partly unknown, it has to be approximated. Several levels of approximation are used for comparison starting from the conventional local density approximation (LDA: PWLDA and SVWN), continuing with a semilocal generalised gradient expansion (GGA: PBE), including meta-GGA using kinetic energy density (TPSS [20,21]) and finishing with orbital dependent KLI [22, 23] and LHF [24] leading to multiplicative potential operator and hybrid B3LYP the potential including the non-multiplicative Fock operator.

Reliability and accuracy of excitation energetics is commonly enhanced by the time-dependent generalisation of DFT . Here, the linear response scheme within the adiabatic approximation [25] is applied, both in LDA and PBE-GGA.

\section{B. Software}

We have chosen to apply the software packages Turbomole, Version 5.7 [26, 27] (to be abbreviated ' $T$ '), Spartan, Release 5.1.1a2 [28] ('S'), Gaussian 94(TM), Revision E.2 [29] ('G') and GPAW, Version 0.9.0 [30, 31] ('P').

$\mathrm{T}$ and $\mathrm{G}$ use Gaussian basis sets, whereas a numerical basis is used in S when DFT is applied.

The real-space approaches, implemented in $\mathrm{P}^{2}$ among some others, offer at least two significant benefits compared to basis function methods: they allow arbitrary boundary conditions (BC), for example flexibly combining Dirichlet and periodic (or other) BCs, and "the potential of massive parallelisation" [30]. On the other hand, finite difference discretisation used in GPAW has the weakness of not being variational: it is an approximation to the operator, not the operand like the basis function methods.

$\mathrm{T}$ and $\mathrm{S}$ are all-electron codes. $\mathrm{P}$ exploits finite difference stencils and the frozen core approximation with 1s-electrons in the core of elements $\mathrm{C}, \mathrm{N}$ and $\mathrm{O}$.

All TDDFT results are obtained by Turbomole.

\section{RESULTS}

\section{A. Molecular Conformation and Electronic Structure}

According to several $a b$ initio calculations presented here, both, trans (Fig. (3)) and cis isomers of DR1 are roughly planar, as already reported by Kurita et al. [19] in case of the parent azobenzene molecule, the latter, nevertheless, significantly less, as can be deduced from distortion of the phenyl hexagons in Fig. (2). Conventional semiempirical methods also attempted here (AM1 and PM3) do not, instead, reproduce the controversial planar conformation, at least not without further empirical information, such as restrictions related to planarity, for example, yielding difficulties in case of cis isomer. The agreement of the conformation with experimetal data is satisfactory (Table 1) with virtually exact agreement with trans and less than $2 \%$ error $^{3}$ with cis isomer. The experimentally observed opening of the $\mathrm{C}-\mathrm{N}=\mathrm{N}-\mathrm{C}$ angle in trans to cis transformation is reproduced (Fig. (2), red dashed reference), underestimated by less than $10 \%$ by LDA, and the opening in the nitro end is expected to dominate by a factor of 1.3 (in Table 1). The typical picture of azobenzenes (Fig. (1) or [32, Figure 1]) is misleading in

\footnotetext{
${ }^{2}$ The name GPAW is an abbreviation for Grid-Projector-Augmented-Wave. PAW [31] is a procedure, an engineering trick, one might say, where the orbitals are linearly transfomed into smoother functions allowing sparcer grid, and thus, efficient evaluation of larger systems. Performance is further improved by some multigrid techniques.

${ }^{3}$ Note the method and basis for the data in (Table I) are PWLDA/SVP. Estimating the basis set error by comparing 11.83 here and 11.78 in (Table II) yields errors less than $2 \%$ wrt exp.
} 

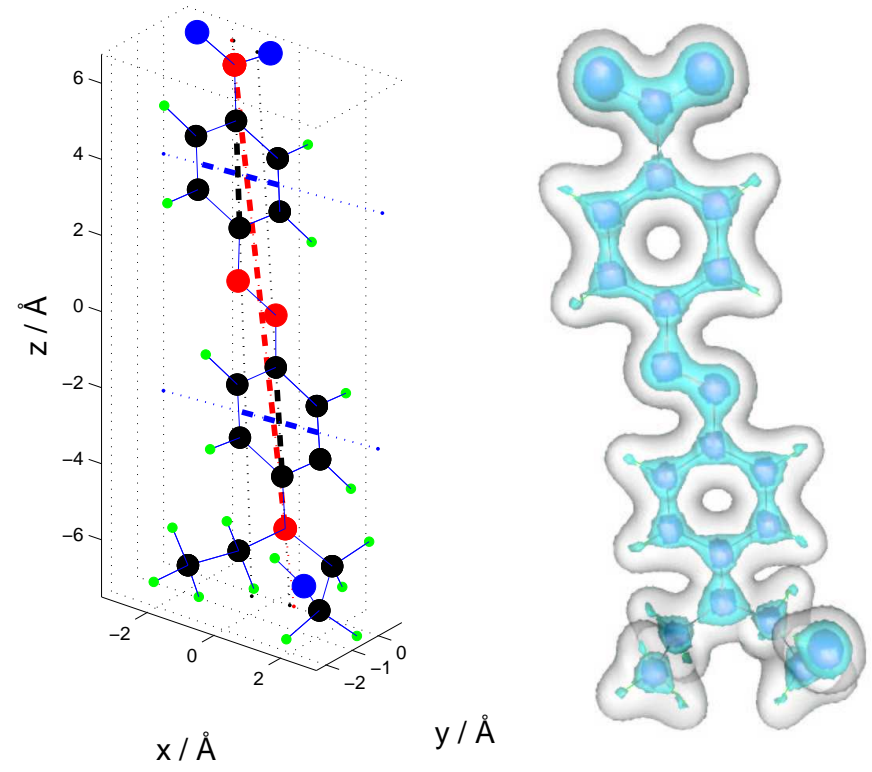

Fig. (3). Optimised PWLDA (see Table 2) conformation of trans1-DR1 (left) by GPAW code (green:H, black:C, red:N, blue:O), see (Table 2) for dashed lines, and ground state electron density isosurfaces (right) $(0.09 \%, 0.46 \%$ and $1.08 \%$ of maximum).

case of DR1 since it strongly exaggerates the opening of C$\mathrm{N}=\mathrm{N}-\mathrm{C}$ angle, as can be seen from Table $\mathbf{1}$ (note $\mathrm{d}_{\mathrm{C}}(\mathrm{C}, \mathrm{C})>\mathrm{d}(\mathrm{C}, \mathrm{C})$ for para carbons in cis isomers). We also note that the cis 2 isomer is more folded than its 1 counter part, where the oxygen atom of the amino ligands is located futher (in the leg 'away') from the nitro group, than in cis2 (see Fig. (2)).

Turbomole ( $\mathrm{T})$ does not support any geometry optimisation for KLI and LHF. ${ }^{4}$ The optimal geometry, within the optimal conformations from the other approximations, corresponds to LDA for KLI and TPSS for LHF (Table 2).

DR1 has a large permanent dipole moment of $8.7 \mathrm{D}^{5}$ (in dimethyl sulfoxide) and it is well known for its large hyperpolarizability [34]. The dipole moment is overestimated by all the methods presented (Table 2), as expected [14], although the magnitude of screening by the polarisation of the solvent is hard to estimate here. The result, however, confirms previous semiempirical estimations [35].

The ground state frontier KS molecular orbitals exhibit strong delocalisation (Fig. (4)) suggesting highly non-linear optical response properties. The HOMO-LUMO and

${ }^{4}$ This is because the geometry optimisation becomes ambiguous in case of approximations directly to the KS-potential, such as KLI and LHF, for which no total energy functional (and thus Hellman-Feynman forces) exists. Some functional integration techniques could be considered [33].

${ }^{5} \mathrm{~A}$ dipole moment strength $8.7 \mathrm{D} \approx 1.8 \mathrm{e} \AA$ corresponds to moving approximately 0.2 electrons from one end of trans-DR1 to the other.
HOMO-(LUMO+1) transitions, however, have low intensity [36] although the orbitals are nearly orthogonal and have spatial overlap. This must be due to mirror symmetry breaking by the amino group and the slight non-planarity of the phenyl rings.

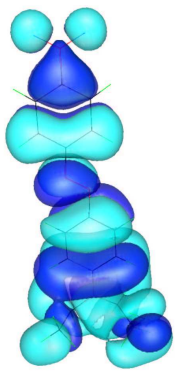

HOMO-1

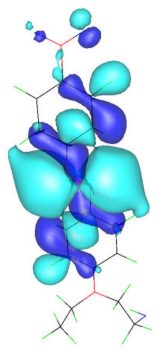

HOMO
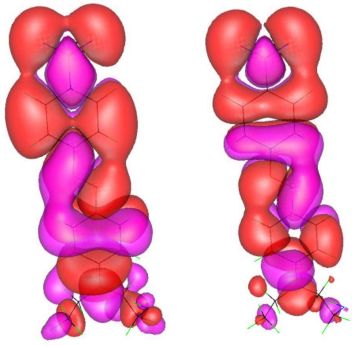

LUMO

\section{$\mathrm{LUMO}+1$}

Fig. (4). Frontier PBE-KS molecular orbitals of trans isomer.

As pointed out above, it would be practical to have available such a one-particle (or non-interacting quasiparticle [37]) picture describing an interacting system, that the eigenenergies produced the excitation spectrum, at least for the lowest excitation. The true KS orbital energies do not yield such. Though KS orbital energy differences of a single SCF run clearly underestimate the transition energies, see Table 2, they nevertheless are often used as a zeroth order approximation. Within HF formalism the difference of total 
energies of two SCF runs should be used, instead, as a better approximation for ${ }^{6}$ the optical gap, whereas this is not the case within DFT. ${ }^{7}$ Comparison of the eigenenergy differences shows that HF (as expected) but also the popular B3LYP fail here with the transition energetics (Table 2). Since DR1 is a rather delocalised system, the latter failure could be associated with the incorrect asymptotic behaviour of B3LYP in the homogenous limit [39, III.C.2].

Table 1. The Structure of DR1 (Turbomole: PWLDA/SVPBasis)

\begin{tabular}{|c|c|c|c|c|}
\hline distance / § & trans 1 & trans 2 & cis 1 & cis 2 \\
\hline $\mathrm{d}(\mathrm{N}, \mathrm{N})^{a}$ & $11.83^{g}$ & 11.83 & 7.25 & 7.18 \\
\hline $\mathrm{d}_{\mathrm{c}}(\mathrm{C}, \mathrm{C})^{b}$ & 9.02 & 9.02 & 6.95 & 6.92 \\
\hline $\mathrm{d}(\mathrm{C}, \mathrm{C})^{c}$ & 9.02 & 9.02 & 5.63 & 5.59 \\
\hline $\begin{array}{c}\mathrm{d}(\mathrm{C}, \mathrm{C}), \text { exp. } \\
{[32, \text { Figure 1] }}\end{array}$ & \multicolumn{2}{|c|}{9.0} & \multicolumn{2}{|c|}{5.5} \\
\hline $\mathrm{d}(\mathrm{N}, \mathrm{N})^{d}$ & 1.264 & 1.264 & 1.331 & 1.331 \\
\hline $\mathrm{d}(\mathrm{N}, \mathrm{N}), \exp ^{h}[19]$ & \multicolumn{2}{|c|}{1.247} & \multicolumn{2}{|c|}{1.253} \\
\hline angle / deg & trans 1 & trans 2 & cis 1 & cis 2 \\
\hline $\mathrm{C}-\mathrm{N}=\mathrm{N}^{e}$ & 114.8 & 115.0 & 119.2 & 119.0 \\
\hline $\mathrm{N}=\mathrm{N}-\mathrm{C}^{f}$ & 116.0 & 115.8 & 119.2 & 118.9 \\
\hline exp. ${ }^{h}[19]$ & \multicolumn{2}{|c|}{114.1} & \multicolumn{2}{|c|}{121.9} \\
\hline
\end{tabular}

${ }^{a}$ the distance between the nitrogens in the nitro and amino groups (see dashed red line in Fig. (3)).

${ }^{b}$ the distance between the furthest phenyl carbons, differs from d(C,C) (for para carbon atoms) only for cis isomer.

cthe distance between the para carbon atoms (bonded to ligand nitrogens)

the distance $\mathrm{N}=\mathrm{N}$.

${ }^{e} \mathrm{C}$ towards nitro end from $\mathrm{N}=\mathrm{N}$.

${ }^{f} \mathrm{C}$ towards aminno end from $\mathrm{N}=\mathrm{N}$.

${ }^{g}$ Compare to Table 2 for basis set error estimation.

${ }^{h}$ The experimental value refers to the 'parent' azobenzene $\mathrm{C}_{6} \mathrm{H}_{5} \mathrm{~N}=\mathrm{NC}_{6} \mathrm{H}_{5}$ only, without the (amino and nitro) ligand groups.

\section{B. Photoabsorption of trans Isomer}

So far, the long lasting goal of DFT beyond ground state properties has been simply to come up with a satisfactory description of energetics. One of the main measures of success of an attempt (an introduced approximative XCfunctional, for example ${ }^{8}$ ) has been the ability to deal with the band gap problem, or the prediction of the correct HOMOLUMO gap in case of molecules, usually underestimated. As expected, and is seen from Table $\mathbf{2}$ some evolution exists, as

\footnotetext{
${ }^{6}$ As an alternative to the approximation of the Koopman's theorem, which approximates the fundamental energy gap $E_{g}:=I-A$ instead of the optical one, to be precise.

${ }^{7}$ Except for some approximations of DFT, such as Hartree-Fock-Slater, where even one SCF is enough by introducing a further approximation called a transition state [38].

${ }^{8}$ Other alternatives may be approximative potentials or something even more imaginary like using the mapping between the KS-orbitals and the density.
}

the HOMO-LUMO difference increases with more advanced approximations for XC-energy. This yields better agreement with the first (forbidden) excitation energy obtained from LRT-TDDFT (see Fig. (5)). This is the case, though not necessarily excpected, also for the estimation of the first observable excitation when KLI is compared to LDA and GGA functionals (Fig. (5)) as the significant orbital energy differences, (HOMO-1)-LUMO and (HOMO-1)-(LUMO+1), increase (Table 2). ${ }^{9}$

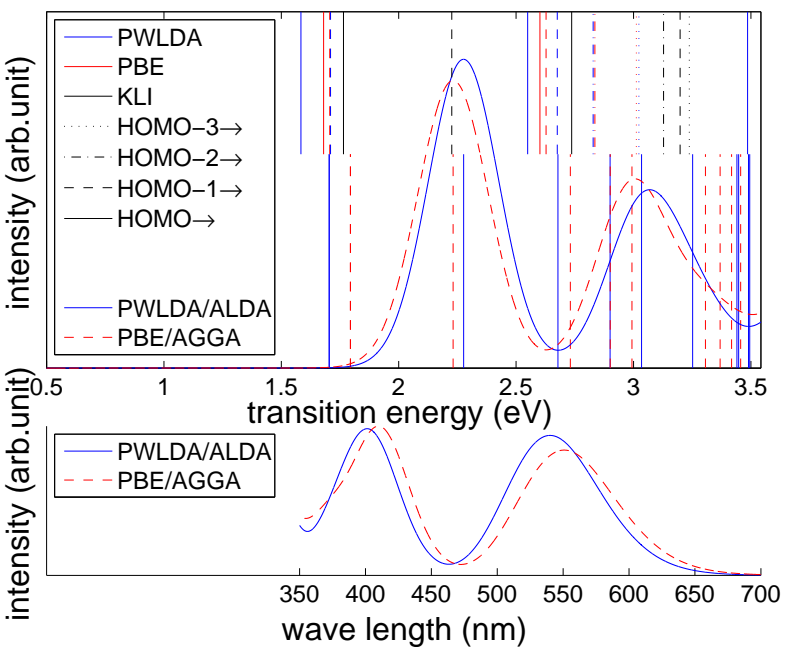

Fig. (5). Comparison of KS-eigenenergy differences and TDDFT transition energies.

TDDFT transition energies for singlet states (top,below) and resulting photoabsorption spectra (top, continuous curve) of trans1 compared to KS-eigenvalue differences (top, above).

TOP, BELOW: Excitation enegies on two stationary XCfunctionals and

TOP, CONTINUOUS: their Gaussian smearing with relative intensities, the first peak width matched with experiment (modelling unhomogenous broadening see Fig. (6)).

TOP, ABOVE: Previous spectra wrt T-PWLDA(blue), PBE(red) and KLI(black) KS-differences-transitions from HOMO(solid), H-1 (dashed), $\mathrm{H}-2$ (dashdot) and $\mathrm{H}-3$ (dotted) KS-states. $0.8 \mathrm{eV} \approx 1550$ $\mathrm{nm}, 3.54 \mathrm{eV} \approx 350 \mathrm{~nm}, 700 \mathrm{~nm} \approx 1.77 \mathrm{eV}$,

BOTTOM: Above photoabsorption spectra as a function of wave length.

TDDFT-LRT is clearly the most accurate method tested here. Not only TDDFT resolves the ambiguity ${ }^{10}$ of interpreting the transitions from HOMO-1 for the singlet states (Fig. (5)), but it does it with quite a low computational extra cost on top of a DFT run. In addition, keeping in mind the finite temperature and effects of the environment present in experimental results (the order of magnitude seen in Fig. (6)) but not in simulations, the less than $10 \%$ accuracy is quite satisfactory (Table $\mathbf{2}$ ).

\footnotetext{
${ }^{9}$ The derivative discontinuity [39], here nonzero only with KLI and LHF, is not included.

${ }^{10}$ Neither transition, (HOMO-1)-LUMO around 1.7-1.9 eV nor (HOMO-1)(LUMO+1) between 2.5-2.9 eV, depending on the XC-approximation, quite agrees with the prediction by TDDFT-LRT. Also, if compared to the experimental $2.5 \mathrm{eV}$, (HOMO-1)-LUMO remains problematic, except for EXX-approximations.
} 
Table 2. The Structure and Energetics of DR1 [40]

\begin{tabular}{|c|c|c|c|c|c|c|c|c|}
\hline METHOD & $\begin{array}{c}\varepsilon_{\mathrm{H}^{-}} \varepsilon_{\mathrm{L}} \\
(\mathrm{eV})\end{array}$ & $\begin{array}{c}\varepsilon_{\mathrm{H}}-1-\varepsilon_{\mathrm{L}} \\
(\mathrm{eV})\end{array}$ & $\begin{array}{c}\varepsilon_{\mathrm{H}}-1-\varepsilon_{\mathrm{L}}+1 \\
(\mathrm{eV})\end{array}$ & $\begin{array}{c}\varepsilon_{\mathrm{H}^{-}} \varepsilon_{\mathrm{L}}+1 \\
(\mathrm{eV})\end{array}$ & $\begin{array}{c}\text { Dipole } \\
\text { (a.u./debye) }\end{array}$ & $\begin{array}{c}\angle 1^{a} / \angle 2^{a} \\
(\mathrm{deg})\end{array}$ & $\begin{array}{c}\mathbf{d}(\mathbf{N}, \mathbf{N})^{b} \\
(\AA)\end{array}$ & code \\
\hline $\mathrm{HF}$ & 8.75 & 10.2 & 12.20 & 10.43 & $3.5 / 8.9$ & $2.4 / 6.1$ & 11.86 & $\mathrm{~T}^{f}$ \\
\hline PWLDA & 1.56 & 1.67 & 2.63 & 2.52 & & $1.4 / 1.7$ & $11.80^{\circ}$ & $\mathrm{P}^{g}$ \\
\hline SVWN & 1.24 & 1.71 & 2.54 & 2.07 & & & & $\mathrm{~S}^{g}$ \\
\hline PWLDA & 1.58 & 1.71 & 2.68 & 2.55 & $4.7 / 12.0$ & $1.4 / 1.4$ & 11.78 & $\mathrm{~T}^{g}$ \\
\hline PBE & 1.68 & 1.71 & 2.63 & 2.60 & $4.7 / 12.0$ & $1.5 / 1.2$ & 11.93 & $\mathrm{~T}^{g}$ \\
\hline TPSS & 1.78 & 1.91 & 2.85 & 2.72 & $4.7 / 12.0$ & $1.6 / 1.5$ & 11.91 & $\mathrm{~T}^{g}$ \\
\hline B3LYP & 2.92 & 3.59 & 4.72 & 4.05 & & $1.4 / 1.3$ & 11.93 & $\mathrm{G}^{g}$ \\
\hline $\mathrm{KLI}^{c}$ & 1.76 & 2.23 & 3.20 & 2.74 & $5.3 / 13.5$ & PWLDA $^{e}$ & $\mathrm{PWLDA}^{e}$ & $\mathrm{~T}^{g}$ \\
\hline $\mathrm{LHF}^{d}$ & 1.88 & 1.99 & 2.84 & 2.95 & $4.6 / 11.8$ & TPSS $^{e}$ & TPSS $^{e}$ & $\mathrm{~T}^{g}$ \\
\hline METHOD $^{h}$ & $\begin{array}{l}\Delta \mathbf{E}_{1} \\
(\mathrm{eV})\end{array}$ & & $\begin{array}{l}\Delta \mathbf{E}_{2} \\
(\mathrm{eV})\end{array}$ & $\begin{array}{l}\Delta \mathbf{E}_{3} \\
(\mathrm{eV})\end{array}$ & & & & code \\
\hline TDDFT(s) & 1.70 & & $2.28^{i}$ & 2.68 & $4.7 / 12.0$ & PWLDA & PWLDA & $\mathrm{T}^{g}$ \\
\hline TDDFT(s) & $1.79^{j}$ & & $2.23^{k}$ & $2.73^{l}$ & $4.7 / 12.0$ & PBE & PBE & $\mathrm{T}^{g}$ \\
\hline TDDFT(t) & 1.44 & 1.60 & 2.57 & 2.45 & $4.7 / 12.0$ & PWLDA & PWLDA & $\mathrm{T}^{g}$ \\
\hline TDDFT(t) & $1.44^{m}$ & $1.54^{n}$ & 2.48 & 2.44 & $4.7 / 12.0$ & PBE & PBE & $\mathrm{T}^{g}$ \\
\hline exp. & $2.5[41]$ & & & & $8.7 \mathrm{D}[14,16]$ & & & \\
\hline ref. & & & & & $12.27 \mathrm{D}[35, \mathrm{AM} 1]$ & & & \\
\hline
\end{tabular}

${ }^{a} \angle 1$ and $\angle 2$ are the angles between dashed black and blue lines (see Fig. (3)), respectively, describing how well the two phenyl rings align in a plane.

${ }^{b}$ see Table 1.

${ }^{c}$ nuclear conformation from PWLDA-QZVPP.

${ }^{d}$ nuclear conformation from TPSS-QZVPP.

${ }^{e}$ The conformation corresponding to the KLI and LHF minimum energies, respectively, within optimal PWLDA, PBE and TPSS conformations is chosen. This is because the geometry optimisation is ambiguous in case of KS-potential approximations for which no energy functional exists [33].

${ }^{f}$ The basis used is TZVPP.

${ }^{g}$ The bases used with T, G and S are QZVPP, 6-31G(d) and DN respectively. P uses $0.2 \AA$ grid spacing.

${ }^{h}(\mathrm{~s})$ and $(\mathrm{t})$ stand for singlet and triplet excitations respectively.

${ }^{i}$ Dominating orbital transitions: homo- $1 \rightarrow$ lumo: $85.3 \%$, homo- $1 \rightarrow$ lumo $+1: 10.5 \%$.

'homo $\rightarrow$ lumo: $74.6 \%$, homo- $1 \rightarrow$ lumo: $24.1 \%$.

${ }_{\mathrm{h}-1} \rightarrow \mathrm{l}: 64.5 \%, \mathrm{~h} \rightarrow \mathrm{l}: 20.7 \%, \mathrm{~h}-1 \rightarrow \mathrm{l}+1: 8.5 \% 9$.

'homo $\rightarrow$ lumo $+1: 76.3 \%$, homo-1 $\rightarrow$ lumo $+1: 22.0 \%$.

${ }^{m}$ homo $\rightarrow$ lumo: $79.1 \%$, homo-1 $\rightarrow$ lumo: $17.5 \%$

"homo-1 $\rightarrow$ lumo: $80.3 \%$, homo $\rightarrow$ lumo: $17.9 \%$.

${ }^{o}$ Compare to Table $\mathbf{1}$ for basis set error estimation.

Measuring the absorption spectrum of the cis isomer is not straightforward because of its thermal unstability. However, the experimental [42] and our estimation agree. It seems tempting to address the experimental data into the joint absorption by trans and cis isomers (Fig. (6)) and refer to low resolution of the experiment to achieve excellent agreement. It would require the amout of $c i s$ and isomers to be at least the same order of magnitude as for trans, unlikely in the light of experimental conditions [9, 17, 41]. The resolution of the measurements is low due to unhomogenous broadening caused by intermolecular interactions.

Since there is one single monomer with Dirichlet boundary conditions in the simulation, there can be no aggregation effects whatsoever. Therefore, the second excitation of trans conformation in Fig. (6) below $400 \mathrm{~nm}$ must not be confused with the experimental absorption in the aggregated system.

LDA/ALDA- and GGA/AGGA-approximations yield essentially the same spectrum at low excitations (Fig. (5)). Although further elimination-e.g. adding results of LDA/AGGA and GGA/ALDA-would be needed, this reflects the general observation that the approximative XCkernel plays a minor role [25] whereas the accuracy of the unperturbed stationary $\mathrm{KS}$-state is quite essential. The accuracy of XC-approximations is not high enough to distinguish the location of the amino-oxygen ('left or right footed'), but clearly recognises the isomer (Fig. (6)).

The real-space grid approach GPAW is able to compete with basis set methods both, in accuracy (Table 2) and in performance. 

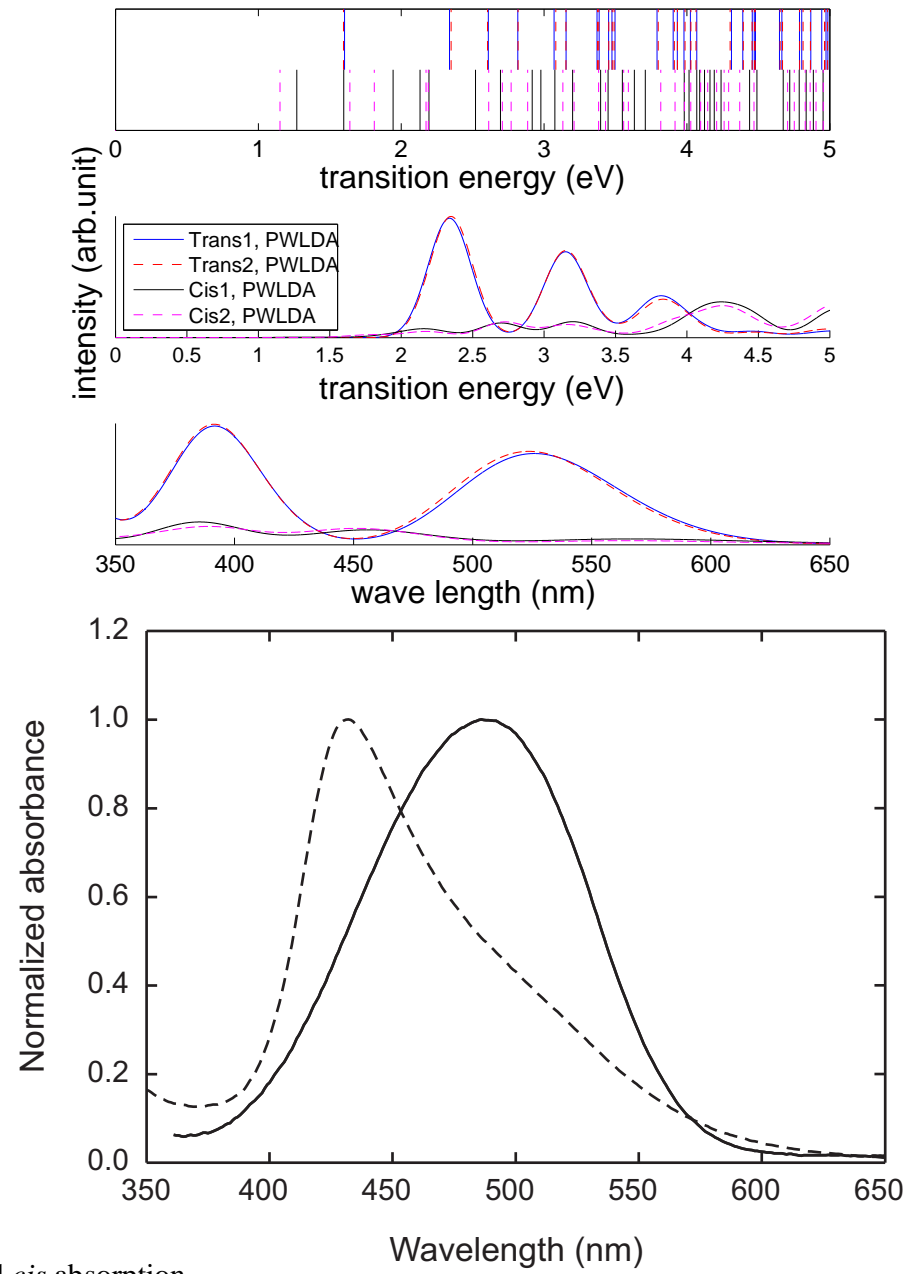

Fig. (6). Comparison of trans and cis absorption.

TDDFT photoabsorption spectrum (three upmost figures, see Fig. (5) for explanation) on LDA- and GGA-KS orbitals of trans1 molecule and on LDA-KS orbitals of cis1 and cis2 molecules compared to experimental data (at the bottom). In the former, the lifetimes of excitations are approximated equal. Thus the relative heights have little or restricted significance. In the experimental part, the solid and dashed lines correspond to the absorption spectra of 'isolated' and aggregated DR1 molecules, respectively. The former is measured in a dilute tetrahydrofuran solution, while the aggregated spectrum is obtained from a highly concentrated (40 wt \%) thin film of DR1 doped in polystyrene.

\section{Photoisomerisation}

In case of DR1, the conformation change resulting from an electronic excitation is significant. The excitation energy leading to the reverse cis-trans photoisomerisation process is some $2.7 \mathrm{eV}$ or around $460 \mathrm{~nm}$, although weak transitions take place already starting from $600 \mathrm{~nm}$ (Fig. (6)), including small contribution also from the HOMO, whereas the barrier for the spontaneous thermal back reaction seems to be of the order of a half of an $\mathrm{eV}(\sim 0.3-0.4 \mathrm{eV})$ (Fig. (7)), and thus would not be easily thermally activated, though sources of error may here be significant ${ }^{11}$. A more probable explanation

\footnotetext{
${ }^{11} \mathrm{We}$ only present a rough estimate for the thermal energy barrier since three sources of error occur. The lack of ligand relaxation (1) overestimates the thermal barrier whereas the fact that only one conformation in the phase space, on a path (2) and at a point on that path (3) chosen by symmetry, is checked may both, increase and decrease the estimation. The chosen path may not be the one on which the lowest pass is found leading to overestimation. The point may not be the highest on that path resulting in underestimation. The path is defined by the bond to be rotated, point by the rotation angle. We believe the points (2) and (3), though easily larger in magnitude than (1), are here not the essential ones, as explained later in the text.
}

for the calculated thermal barrier for the conformation change is that, referring to experiments according to which the thermal barrier is negligible already at room temperature $[4,43]$, the non-planarity of phenyl rings is significant in the energetics aready at half way in the path of rotating the $\mathrm{N}=\mathrm{N}$ bond. The apparent non-planarity of the cis conformation (Fig. (2)) is reflected by the above mentioned less strict selection rule concerning transitions from HOMO. Also, dispersive forces due to long range electronic correlations in the cis conformation are expected to be more significant though nevertheless negligible both, because of more folded structure than in case of trans isomer, and the reduced ground state dipole moment.

Absorption in a cis form shifts the dominant peak to UV range (Fig. (6)). The location of the oxygen atom in the amino group has no significant effect on low cis-absorption, and neither in the trans-cis process.

\section{Dimerisation}

A variety of orientations of two interacting trans-DR1 molecules were scanned with PWLDA approach. The 


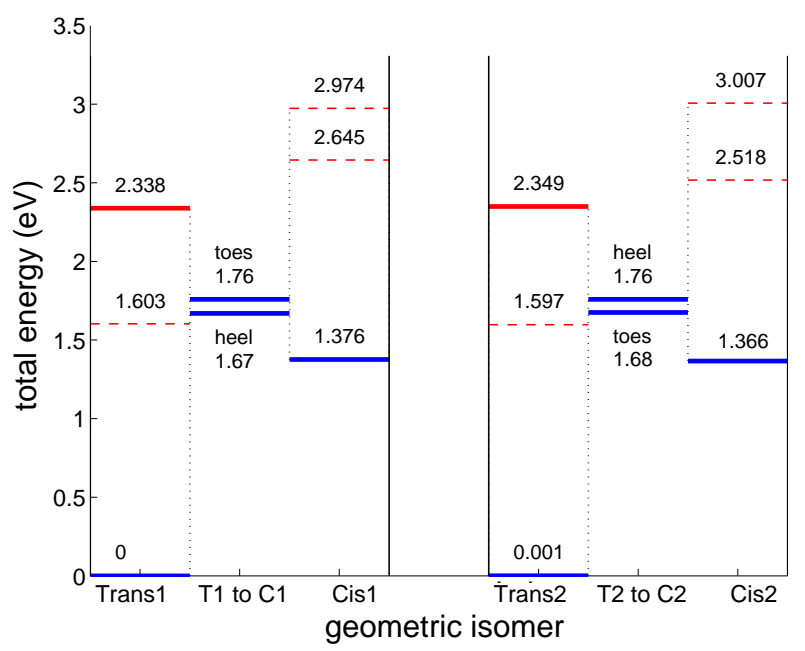

Fig. (7). Trans-cis energetics (by PWLDA in Turbomole): ground and excited states, with blue and red respectively (the almost forbidden transitions dashed). Isomers $\mathrm{T} \rightarrow \mathrm{C}$ are obtained by twisting the corresponding trans form by $\pi / 2$ rad wrt the $\mathrm{N}=\mathrm{N}$ bond. They provide an estimate for the thermal energy barrier, since the lack of ligand relaxation increases the barrier whereas the fact that only one conformation in the phase space, though chosen by symmetry, is checked may underestimate the energy. 'Toes' stands for rotating the nitro end of the molecule clockwise ('righthandside' in Fig. (2)) and 'heel' vice versa. See footnote 1.

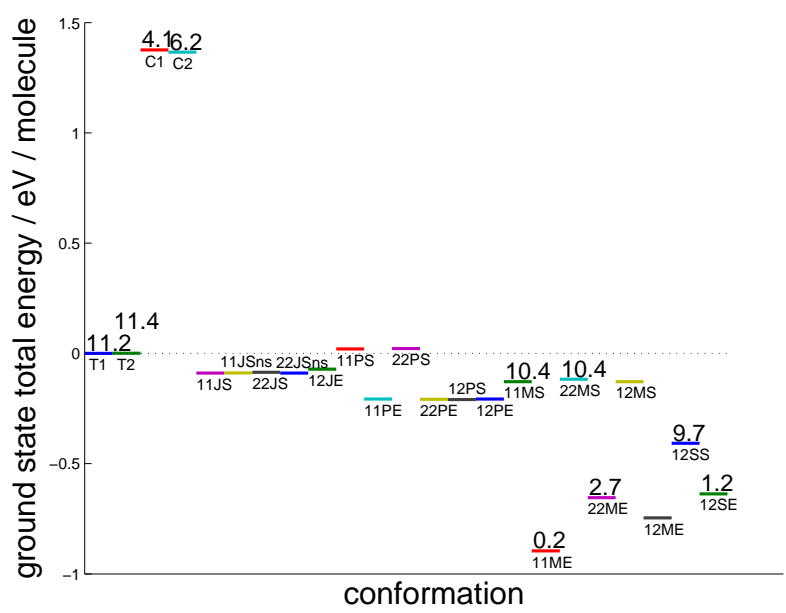

Fig. (8). Ground state total energies and dipole moments (in debyes, by PWLDA in Turbomole) of some trans dimer conformations compared to those of single molecules. (Note in comparison with Table 2, the method and basis for the data here are PWLDA/SVP.) The first letter J, P, M or S stands for 'foot', 'head', 'stomach' or 'back' contact of the monomers. See footnote 1. The second letter S or E refers to parallelity or antiparallelity of the molecular figure, respectively, from which (with Fig. (9)) the dipole effect is obvious. The conformations MS and SS are commonly referred to as J-aggregates whereas the rest are called H-aggregates.

ground state total energies (Fig. (8)) show that dimerisation, as a first step towards aggregation processes, but also in trans-cis photoisomerisation process can be relevant. Several dimers, e.g. "11ME" (Fig. (9)), are energetically more stable (of the order of an $\mathrm{eV}$ per molecule) than a single molecule. This provides a guideline in assessing the relevance of aggregation in solvents. The dimerisation is obviously enhanced by the strong dipole moment of the trans molecule (the "ME" cases). Because of the dipole dominance, no DFT-related trouble with van der Waals effects is expected, although long range electron correlation already at intramolecular level may be relevant in systems of this size. 

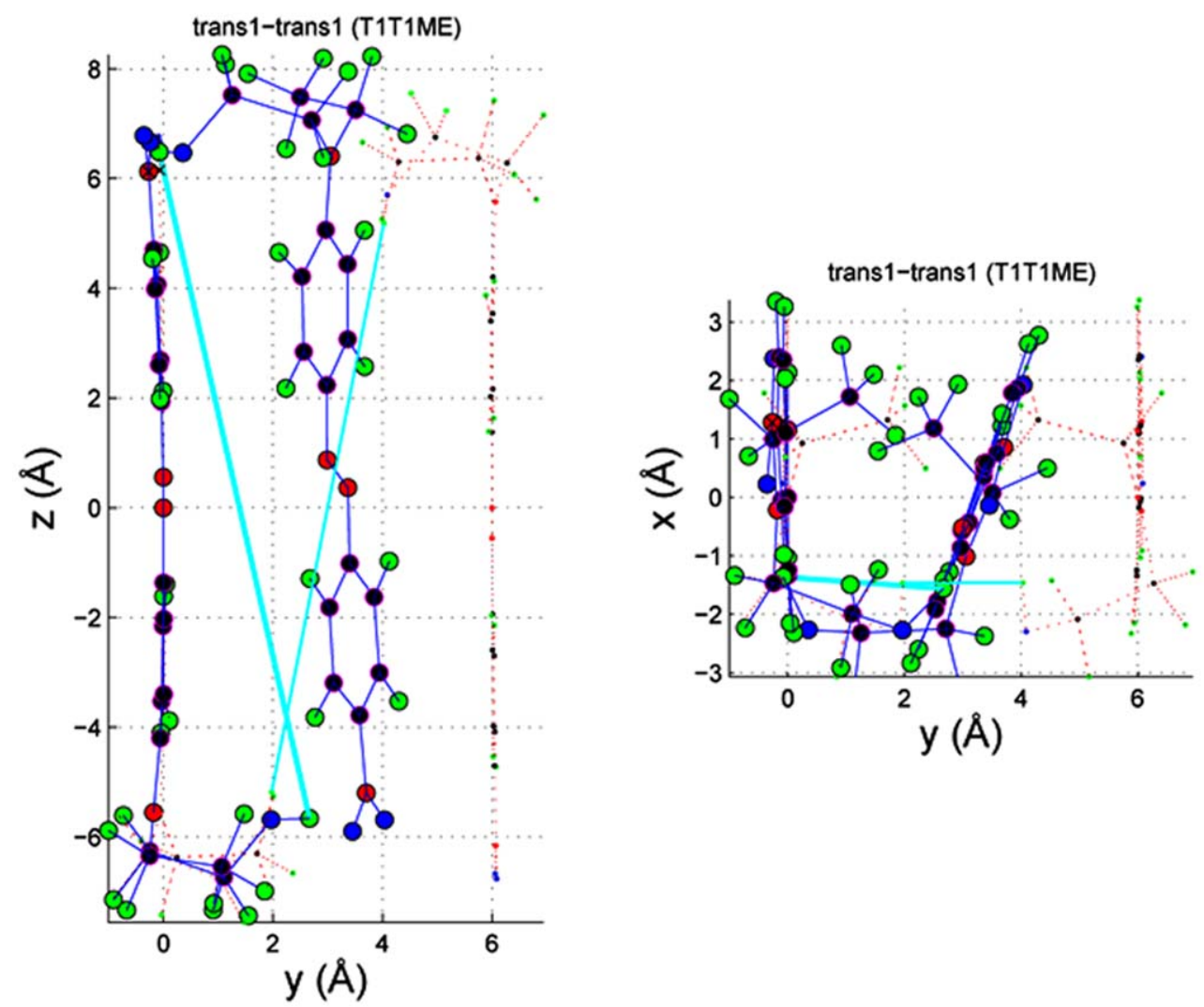

Fig. (9). A local minimum energy conformation of a trans1-trans1 dimer (11ME in Fig. (8)). Cyan lines ease comparing the side (left) and top (right) views. Red lines are the initial guess. (by PWLDA in Turbomole).

The only dimers here with monomer dipoles in the same direction called MS and SS, more commonly referred to as "parallel J-aggregates", shift the absorption peaks towards blue whereas "H-aggregates" appear to cause redshift (Fig. (10)), in agreement with experimental data [44]. ${ }^{12}$

The somewhat suprisingly low total energy of J-dimer 12SS (Fig. (8)) is explained by diminished dipole moment compared to $11 \mathrm{MS}$ and 22MS. The same classical argument does not, however, apply to $11 \mathrm{ME}, 22 \mathrm{ME}$ and $12 \mathrm{SE}$, where the classical dipole-dipole energy is negative, on the contrary to previous, whence decreasing the dipole should increase the energy.

The dimerisation can affect the photoisomerisation barrier by lowering the ground state energy and shifting the photoabsorption peaks. For example, in case of 11ME-dimer, the first observable transition (Fig. (10)) is not high enough to overcome the ground state of the cis isomer (Fig. (7)) and thus dimerisation even prevents photoisomerisation via this transition.

\section{CONCLUSION}

According to the total energy electronic structure calculations Disperse Red 1 takes closely planar

\footnotetext{
${ }^{12}$ In homomolecular dimers it is irrelevant whether the terms $\mathrm{H}$ and $\mathrm{J}$ refer to stationary or transition dipole moments (diagonals and off-diagonals respectively). The key question is, are the dipoles in-line, or parallel as here.
}

conformation in both of its isomers, trans and cis with possibly significant breakup of symmetry in case of cis though. Consequently, the frontier orbitals become conjugated and delocalised in the whole molecule. This is consistent with the experimentally observed highly nonlinear optical properties, which however, is not the subject of the present study. Here, we have concentrated on the lowest photoabsorption transitions, and in particular, comparison of the performance of DFT related approaches in light of the interpretation of experimental spectra.

First, we find that the dipole transition matrix element of HOMO and LUMO orbitals is small due to their symmetries. Nevertheless, the lowest observable energy excitation is essentially from HOMO-1 to LUMO. In fact, only a few of the one-electron orbitals contribute to the photoabsorption, and furthermore, the cis isomer presents weaker absorption of the two in the visible range. As expected, the Kohn-Sham eigenvalue differences from LDA and GGA underestimate the HOMO-LUMO gap, and thus, the lowest forbidden photoabsorption. Interpreting higher transitions leads to ambiguities resolved by TDDFT. The TDDFT excitations energies match best with the experimentally observed absorption bands. The conventional GGA does slightly better work than LDA and inclusion of non-local exchange improves transition energies even further. However, as expected in a delocalised system, use of B3LYP functional leads to clear overestimation in this case, probably indicating too strong contribution from Hartree-Fock exchange. 


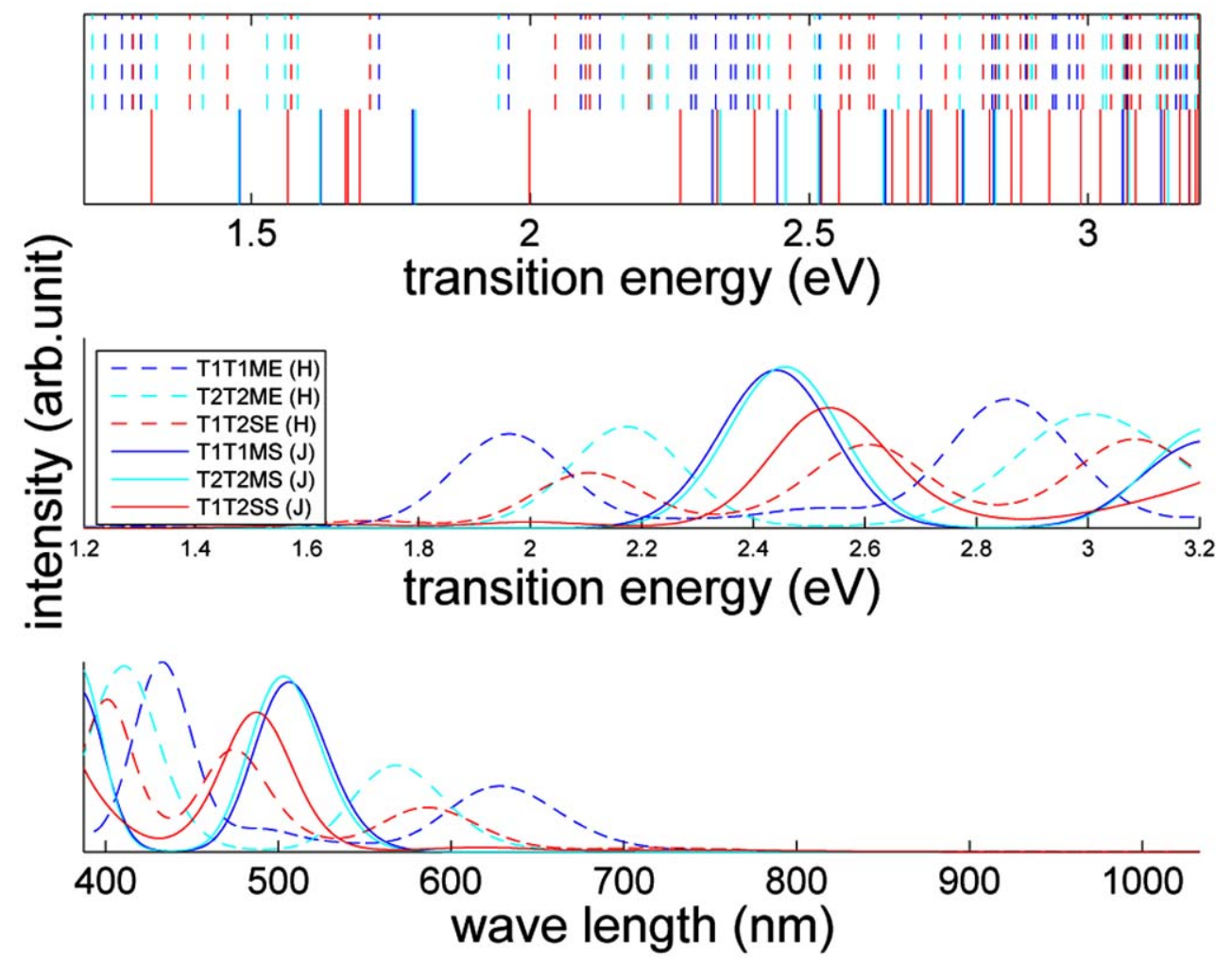

Fig. (10). Photoabsorption of dimers.

TDDFT photoabsorption spectrua (see Fig. 5 for explanation) on LDA-KS orbitals (SVP basis) of trans dimers. The lifetimes of excitations are approximated equal. Thus the relative heights have little or restricted significance.

The cis isomer of DR 1 has about $1.4 \mathrm{eV}$ higher total energy than its trans counterpart. Due to the large dipole moment of trans isomer dimerisation can be expected, in a nonpolar solvent in particular. In vacuum we find the dimerisation energy per molecule to be upto $0.9 \mathrm{eV}$.

\section{ACKNOWLEDGMENTS}

The authors thank Arri Priimägi for his essential contribution in experimental connections, the Nonlinear optics group of TUT for their interest and fruitful discussions and CSC for computational facilities. The first author acknowledges 'ampereen kaupungin tiederahasto' and the National Graduate School of Materials Physics for financial support.

\section{REFERENCES}

[1] Delaire JA, Nakatani K. Linear and nonlinear optical properties of photochromic molecules and materials. Chem Rev 2000; 100: 1817-46.

[2] Matharu AS, Jeeva S, Ramanujam PS. Liquid crystals for holographic optical data storage. Chem Soc Rev 2007; 36: 186880.

[3] Kanis DR, Ratner MA, Marks TJ. Design and construction of molecular assemblies with large second-order optical nonlinearities. Quantum chemical aspects. Chem Rev 1994; 94: 195-242.

[4] Rau H. Photoreactive Organic Thin Films. In: Sekkat Z, Knoll W, Eds. San Diego, USA: Academic Press 2002.

[5] Bosshard C, Sutter K, Prêtre P, et al. Organic nonlinear optical materials. Basel: Gordon and Breach Science Publishers SA 1995.

[6] Natansohn A, Rochon P. Photoinduced motions in azo-containing polymers. Chem Rev 2002; 102: 4139-76.
[7] Yesodha SK, Pillai CKS, Tsutsumi N. Stable polymeric materials for nonlinear optics: a review based on azobenzene systems. Prog Polym Sci 2004; 29: 45-74.

[8] Blau W. Organic materials for nonlinear optical devices. Phys Technol 1987; 18: 250-61.

[9] Priimägi A, Cattaneo S, Ras RHA, Valkama S, Ikkala O, Kauranen M. Polymer-dye complexes: a facile method for high doping level and aggregation control of dye molecules. Chem Mater 2006; 17: 5798-802.

[10] Priimägi A, Kaivola M, Rodriguez FJ, Kauranen M. Enhanced photoinduced birefringence in polymer-dye complexes: Hydrogen bonding makes a difference. Appl Phys Lett 2007; 90: 121103.

[11] Dalton LR, Harper AW, Robinson BH. The role of London forces in defining noncentrosymmetric order of high dipole moment-high hyperpolarizability chromophores in electrically poled polymeric thin films. Proc Natl Acad Sci 1997; 94: 4842-7.

[12] Zaleśny R, Matczyszyn K, Kaczmarek A, Bartkowiak W, Cysewski P. Experimental and theoretical investigations of spectroscopic properties of azobenzene derivatives in solution. $\mathbf{J}$ Mol Model 2007; 13: 785-91.

[13] Briquet L, Vercauteren DP, André JM, Perpète EA, Jacquemin D. On the geometries and UV/Vis spectra of substituted transazobenzenes. Chem Phys Lett 2007; 435: 257-62.

[14] Wang YJ, Carlisle GO. Optical properties of disperse-red-1-doped nematic liquid crystal. J Mater Sci-Mater El 2002; 13: 173-8.

[15] Sek D, Schab-Balcerzak E, Solyga M, Miniewicz A. Polarizationsensitive holographic recording in polyimide-containing azo-dye. Synth Met 2002; 127: 89-93.

[16] Pliška T, Cho WR, Meier J, et al. Comparative study of nonlinearoptical polymers for guided-wave second-harmonic generation at telecommunication wavelengths. J Opt Soc Am B 2000; 17: 155464.

[17] Egami C, Suzuki Y, Sugihara O, et al. Third-order resonant optical nonlinearity from trans-cis photoisomerization of an azo dye in a rigid matrix. Appl Phys B 1997; 64: 471-8.

[18] Sekkat Z, Wood J, Aust EF, Knoll W, Volksen W, Miller RD. Light-induced orientation in a high glass trnsition temperature 
polyimide with polar azo dyes in the side chain. J Opt Soc Am B 1996; 13: 1713-24.

[19] Kurita N, Tanaka S, Itoh S. Ab initio molecular orbital and density functional studies on the stable structures and vibrational properties of trans- and cis-azobenzenes. J Phys Chem A 2000; 104: 8114-20.

[20] Tao J, Perdew JP, Staroverov VN, Scuseria GE. Climbing the density functional ladder: nonempirical meta-generalized gradient approximation designed for molecules and solids. Phys Rev Lett 2003; 91: 146401.

[21] Perdew JP, Tao J, Staroverov VN, Scuseria GE. Meta-generalized gradient approximation: explanation of a realistic nonempirical density functional. J Chem Phys 2004; 120: 6898-911.

[22] Krieger JB, Li Y, Iafrate GJ. Construction and application of an accurate local spin-polarized Kohn-Sham potential with integer discontinuity: exchange-only theory. Phys Rev A 1992; 45: 101-26.

[23] Krieger JB, Li Y, Iafrate GJ. Systematic approximations to the optimized effective potential: application to orbital-densityfunctional theory. Phys Rev A 1992; 46: 5453-8.

[24] Della Sala F, Görling A. Efficient localized Hartree-Fock methods as effective exact-exchange Kohn-Sham methods for molecules. J Chem Phys 2001; 115: 5718-32.

[25] Fiolhais C, Nogueira F, Marques MAL, Eds. A primer in density functional theory, Lecture Notes in Physics. Heidelberg: Berlin, Springer-Verlag 2003; vol. 620.

[26] Ahlrichs R, Bär M, Häser M, Horn H, Kölmel C. Electronic structure calculations on work-station computers: the program system TURBOMOLE. Chem Phys Lett 1989; 162: 165-9.

[27] Eichkorn K, Treutler O, Öhm H, Häser M, Ahlrichs R. Auxiliary basis sets to approximate Coulomb potentials. Chem Phys Lett 1995; 242: 652-60.

[28] Wavefunction Inc., 18401 Von Karman Avenue, Suite 370, Irvine, CA 92612 U.S.A. Available from:http: //www.wavefun.com/

[29] Frisch MJ, Trucks GW, Schlegel HB, et al. Gaussian 94, Revision E.2. Gaussian, Inc., Pittsburgh PA, 1995.

[30] Mortensen JJ, Hansen LB, Jacobsen KW. Real-space grid implementation of the projector augmented wave method. Phys Rev B 2005; 71: 035109.

[31] Blöchl PE. Projector augmented-wave method. Phys Rev B 1994; 50: 17953-79.
[32] Kumar GS, Neckers DC. Photochemistry of azobenzene-containing polymers. Chem Rev 1989; 89: 1915-25.

[33] van Leeuwen R, Baerends EJ. Energy expressions in density functional theory using line integrals. Phys Rev A 1995; 51: 170-8.

[34] Singer KD, Sohn JE, King LA, Gordon HM, Katz HE, Dirk CW. Second-order nonlinear-optical properties of donor- and acceptorsubstituted aromatic compounds. J Opt Soc Am B 1989; 6: 133950 .

[35] Kim TK, Son YA. Affinity of disperse dyes on poly(ethylene terephthalate) in non-aqueous media. Part 2: effect of substituents. Dyes Pigments 2005; 66: 19-25.

[36] Åstrand PO, Ramanujam PS, Hvilsted S, Bak KL, Sauer SPA. Ab initio Calculation of the Electronic Spectrum of Azobenzene Dyes and Its Impact on the Design of Optical Data Storage Materials. J Am Chem Soc 2000; 122: 3482-7.

[37] Mattuck RD. A Guide to Feynman Diagrams in the Many-Body Problem. $2^{\text {nd }}$ ed. New York: Dover Publications, Inc.; 1976.

[38] Dreizler RM, Gross EKU. Density functional theory-an approach to the quantum many-body problem. Berlin, Heidelberg: SpringerVerlag 1990.

[39] K"ummel S, Kronik L. Orbital-dependent density functionals: Theory and applications. Rev Mod Phys 2008; 80: 3-60.

[40] Ojanen J. Elektronien vuorovaikutusten mallintaminen tiheysfunktionaaliteoriassa. Diploma theses, Tampere University of Technology, Finland 2005.

[41] Priimägi A. Nonlinear Optical investigation of self-assembled polymer systems. M.Sc. theses, Tampere University of Technology, Finland, 2004.

[42] Loucif-Saïbi R, Nakatani K, Delaire JA, Dumont M, Sekkat Z. Photoisomerization and second harmonic generation in disperse red one-doped and -functionalized poly(methyl methacrylate) films. Chem Mater 1993; 5: 229-36.

[43] Barrett C, Natansohn A, Rochon P. Cis-trans thermal isomerization rates of bound and doped azobenzenes in a series of polymers. Chem Mater. 1995; 7: 899-903.

[44] Kasha M, Rawls HR, El-Bayoumi MA. The exciton model in molecular spectroscopy. Pure Appl Chem 1965; 11: 371-92. 